subsequent postpartum depression, the case for specificity is rather more compelling. Indeed, the respective age of those with a previous psychiatric history and those without such a history becomes irrelevant when the critical comparison being made is the relative risk at postpartum and nonpostpartum periods. Indeed, if those with a previous history do represent a more severe group, as Bell and colleagues suggest, it is of even more note that they are not at risk for subsequent postpartum depressive episodes.

Winnicott Research Unit

P. J. COOPER

L. MURRAY

Cambridge CBI 2ES

\section{Personality features in chronic depressive episodes}

SIR: It is unfortunate that Brown et al (BJP, October 1994, 165, 447-56 and 457-65) did not examine the role of personality disorder more exhaustively in their report and studies of chronic depressive episodes. Personality status no longer needs to be assessed by 'a crude rating of predominant personality disorder backed up by inter-rater reliability' of unspecified information. There are several instruments that can be used to record the presence of DSM personality disorders more satisfactorily and, although we acknowledge their imperfections, they are undoubtedly superior to the techniques used in this study. It is also accepted that there is often particular difficulty in disentangling the effects of personality from those of chronic illness and even more care with assessments is needed under such conditions. The absence of satisfactory information about personality status is doubly unfortunate when so many other measures are recorded that are often associated with personality disorder. These include life events and early experiences such as sexual abuse, parental separation and delinquency.

The finding of only a marginal association of personality disorder with chronicity in depression is also at variance with most other studies. Although we acknowledge the methodological problems of assessing personality status when there is coexisting mental illness, it is only when workers of stature such as George Brown address these issues squarely that the complex inter-relationship between related life events, childhood adversity and current interpersonal difficulties will be resolved. It is hoped that other investigators will examine personality status in more detail in further studies. Our suspicion is that a careful recording of early childhood experiences and that of current personality status would identify remarkably similar groups of people.

\section{St Charles' Hospital \\ London W10 6DZ}

P. TYRER

University of Manchester

N. SeIVEWRIGHT

Chinese whispers and the prophylactic effect of cognitive therapy

SIR: In Psychotherapy in the maintenance treatment of depression (BJP, December 1994, 165(suppl), 42-50), Weissman summarises evidence for the maintenance and prophylactic effects of cognitive therapy (CT). She states that "results of follow-up studies in patients who have received CT have been mixed". Results have indeed varied in terms of whether they were (Evans et al, 1992) or were not (Shea et al, 1992) significant. However, they have not varied in the direction of effects which have consistently favoured CT over treatment with medication alone.

In addition, when considering evidence suggesting prophylactic effects of CT, Weissman reiterates a conclusion from Shea et al (p. 786) that "The possibility that CT selects healthier patients with a lower latent risk of relapse compared with those recovering with tricyclic antidepressants is an equally plausible explanation". In neither the NIMH study (Shea et al) nor that of Evans et al was there any evidence that differential retention of patients during acute treatment biased the comparison of outcomes over follow-up. Differential retention is particularly unlikely to explain the difference in relapse between patients treated with combined cognitive therapy and pharmacotherapy versus patients treated with pharmacotherapy alone in the Evans et al study. Furthermore, in both of these studies, patients initially treated with cognitive therapy were found to have received less further treatment during follow-up than patients in the other treatment groups. This would be likely to result in an underestimate of any advantage of CT over pharmacotherapy in the prevention of symptomatic relapse without such further treatment. Thus, it could be argued that there is no evidence that the studies overestimate, and some evidence that they may underestimate, the prophylactic effects of cognitive therapy.

That CT prevents relapse of depression beyond termination of therapy has yet to be proved conclusively. However it is important that encouraging 